\title{
Infalt.
}

Mbtürzนngen .

\section{A. Einleitung.}

B. Burgerlides (Befehbud.

(ETjtes $\mathfrak{B}$ ư).

\section{allgewriuet êheil.}

811. פohnfit . . . . . . . . . . . . 7

$\$ 204$. Đerjährung zroffden (Eltern unb Rinbern . \&

3weites Bu(

\section{Sent ver Shulbuerbältuifte.}

$\S 825$. Sकabenseria bet Berlettung zur aufer:

egellden Betwohnung. . . . . . . . . 9

$\$ 5842,847$. ummang bes sdabenderjałes. . . . 10

Bierteร Buฒ.

fanilienredt.

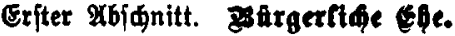

Erfter Titel. Berlöbuif.

$\$ \$ 1300,1302$. Sdabenser|a wegen Betwohnung unter Berlobten . . . . . . . . . . 11 
Gette

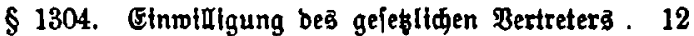

$\$ \S 1305-1308$. Clterlide Etnwialgung . . . . 13

$\S 1310$. EGebinbernif ber Berroanbtfaaft unb

ङฌmägerfळajt. Affinitas illegitima . . . 17

$\$ \S 1311,1314$. Meltere Egefinberniffe . . . . 18

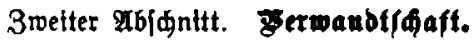

Eriter Titel. Mugemeine Borforiftem.

$\S 1589$. Begriff ber Berroanbtfdaft . . . . . 19

§1590. Begriff ber डdmäger\{ळajt . . . . . 21

3reiter Titel. Cyelidge abftammung.

$\$ \S 1591,1592$. Begriff . . . . . . . . . 22

$\$ \S 1593-1597$. Geltenbmadung ber uneheltdfett.

Infechtung . . . . . . . . . . . . 24

$\$ \S 1599,1599$. 2Tnertennung ber Egelthfeit. An=

fedtung biefer Interfenmung . . . . . . 30

$\S 1600$. Bateridaft im Falle frühyeltiger Bteber= vergetratgung . . . . . . . . . . 31

Dritter Titel. unuterhaltspplitidt.

$\S \S 1601-1615$. . . . . . . . . . . 32

Bierter Titel. Medtlidfe Stellnug ber egefliden Sinder.

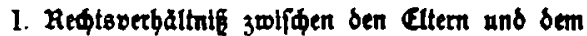
finde in 2llgemeinen. 
Sripalt.

VI

II. Cltetlide bemalt.

\section{Bette}

1. CIterlibe Betwalt we Daters.

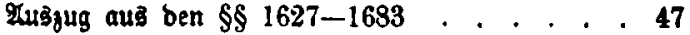

2. Clterltice Belwalt wer shuttur.

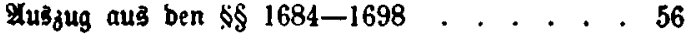

Fürfter Titel. Medtlide Stellnug ber Sinber ans niditigen Chen.

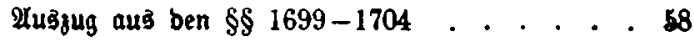

巨echfter Titel. Medtlide Stellung ber uns ebeliden Sinber.

$\S 1705$. Stellung zuะ 9hutter unb zu ben miltter=

llder Berwandter . . . . . . . . . . 61

$\S 1706$. jamiltenname bes̀ Stndeș . . . . . 63

$\$ 1707$. Elterlide (8ewalt. Fedte ber Mutter unb

bes Bormunbs betreffs ber Sorge für bte

Berion . . . . . . . . . . . . . 66

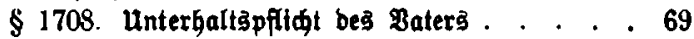

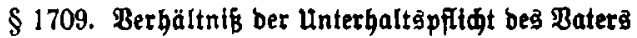

zu berjenigen ber Dutter unb ber mütteritien

Derwanbten . . . . . . . . . . . . 71

\$1710. Belbrente als untergalt. Borauşbejah:

lung . . . . . . . . . . . . . . 72

$\S$ 1711. Unterhalt für bie Bergangenget . . . 75

$\$ 1712$. Tob bes gaters. Abfinbung mus bem

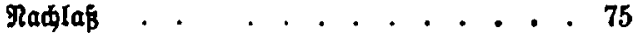

\$1713. Tob bes Rinbes. Beerbtgungälofien . . 77 
1714. Pereinboung aber ben unterbalt

\$ 1715. Anfpridje ber Mutter gegent ben Bater . 79

$\S 1716$. Siderung ber P्Aniprüde vor ber (Seburt 81

$\$ 1717$. Beweis ber Bateridaft. Empfängnifizett 84

$\$ 1718$. Anertennung ber Baterfiaft. . . . 86

Slebenter Titter. Regitimation unehefider Simber.

I. Segiltmatton ourd nadfolgende Ebe.

\$1719. Degrtff . . . . . . . . . . . . 88

$\$ 1720$. Pewets unb Anerfennung ber Bateridaft 90

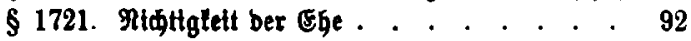

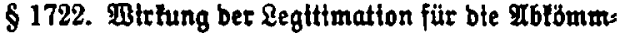

Itnge bes Sitnbes. . . . . . . . . . . . . 92

II. Ebellditelsertlärung.

$\S \S 1723,1724$. Begztf, 3uftänbtglett, 3ngalt . 93

$\$ \$ 1725,1726$. Wntrag bes Waters. Etrrotultgung

ber Betgetltgten . . . . . . . . . . 94

\$1727. Erfełung ber multterliden Einwlatgung 96

$\$$ 1728. Bertretung von Bethelligten . . . . 97

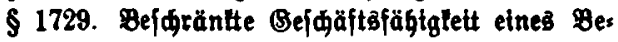

thetilgten. . . . . . . . . . . . . . . 98

$\$ \$ 1730,1731$. Form ber Ertlärungen. 2nfedtung 99

\$ 1732. Egemtbrtge Ainber. . . . . . . . . 99

5 1733. Fob bes Rinbes ober beş \$aterg vor ber

Egelidłettsertlarung . . . . . . . . . 100

\$ 1734. Derfagung ber Egelíblettsertlärung . . 101 
Switalt.

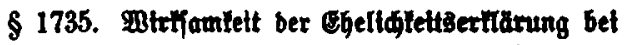

Gette

Shängeln in ben gefelltiden Porausfełungen 101

$\S 1736$. Wirfung ber Ebeltwitettertiarung fiur bas

stinb . . . . . . . . . . . . . . 101

\$ 1737. Bitlung für anbere Famtlienmitglieber 102

$\S 1738$. Wirtung für ble פRutter . . . . . . 103

$\$ 1739$. Wtrtung auf bte unterbaltşpflitht . . 104

$\S 1740$. Egheidlteß̧ung bes \$aters . . . . . 104

Mmter Titel. Sunahme an Sinbesftutt.

$\$ \S 1741-1772$. . . . . . . . . . . . 105

Dritter 2rbidnitt. \$ormxub/áaft.

Griter Titel. Bormunbihaft thber Miuber:

jährige.

I. Unotonung det Dormandfकaft.

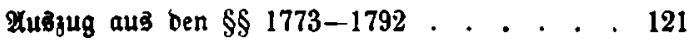

II. fubang der Dotmandjळaf?.

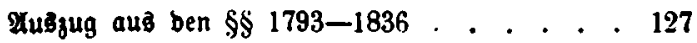

III. fürjorge und 2lufint bes Dormundjạafte. getríts.

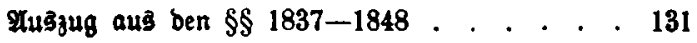

VII. Betnolgung det Dotmundihaft.

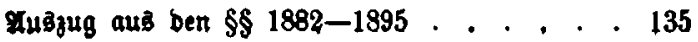


3weiter Titel. Bormunbidnaft Hber Boll= Belte jährige.

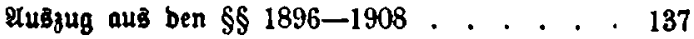

Dritter Tttel. Priegidafit.

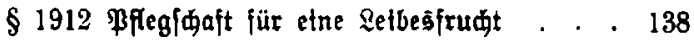

Fünftę̧ Bud).

erturedt.

Esfter Ab/dnttt. Erbfolge.

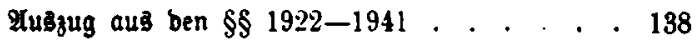

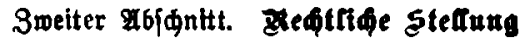

Des Erben.

\$1963. Unterbaltzaniprú ber Dhutter bet zu er= wartenber Beburt etnea Erben . . . . . 145

\$1969. Unterhaltsaripruch von Famtlienange: Görtgen . . . . . . . . . . . . 146

Dritter $2 b \mid$ f)nitt. Teffament.

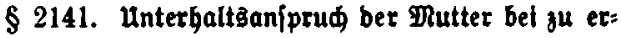
wartenber Beburt eines Raherben . . . . 147

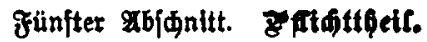

2303. Inโprud auf ben Pflidttgetl . . . 148 


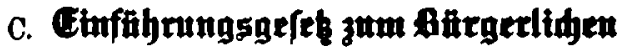
Berethudue.

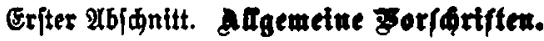

Art. 18, 19. Anwenbung ber beut[den Bejese uber egeltale Abitammung unb über Mechts: vergältntffe ber eheliden Sinber in inter: nationalen Redtsōvertegr . . . . . . . 149

Xrt. 20, 21. Inwertbung ber beutfden unb auşs

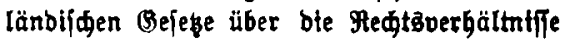
ber unebeliden \$inber . . . . . . . . 150

Rrt. 22. Innenbung ber beutiden Gejełe über Legittmation und Annabme an Sinbeziftatt 151

art. 28-30. Dejonbere Boriditften bes interna* ttonalen Prtwatredts

Drttter 2Ibidnttt. Ferbaftut

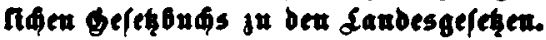

Art. 134. Feligtöle Erzlegung ber Sinber . . . 153

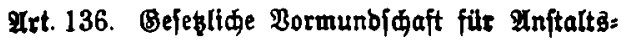
pfleglinge unb unebelithe Rinber . . . . 153

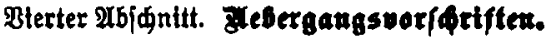

2itt. 208. Stellung ber unegeltwen Rtnber. . . 155

2rt. 209. Stellung ber legitimtaten unb ber an Sinbesfiatt angenommenen stmber . . . 157 
D. Anlagen.

I. Pte Eeftumungen ber \&rojefigefege aber bie serfofgung bes zuteriafts. ax/pruds.

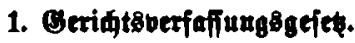

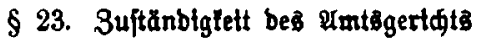

2. Civilprozeforbunng.

\$ 258. Slage auf tünftige Reiftungen . . . . 159

$\S 323$. Zbänberung redtałträftiger Urthetle bes treffenb mieberfegrenbe Retifungen... . 160

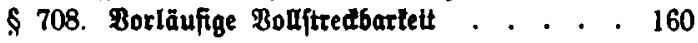

$\$ 850$. Forberungen, bie ber $\mathfrak{P}$ fănoung nidt unterworfen fint . . . . . . . . . 161

3. Befet, betrefferb bie Befiflagrahme bes Arbeits: ober Dienftlotues.

$\S \S 1,4,4 a$

4. Roulur8orbuning.

§ 3. Bebanblung ber unterbaltšs unb ägnlther Injprüde . . . . . . . . . . 165

I. Ausjug aus ben prenfit/on aus-

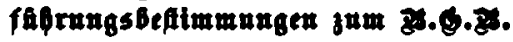

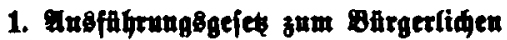
Cefetbad.

2rt. $68 \$ 2,3$. Extlärungen über ben gramiltien: namen 
Suspart.

XII

9itt. Bethe

Irt. 70. Fnetlennung bex Baterføaft . . . 167

Frt. 71. Befonbere Borfartft flix bas rhetulfoc Reft. . . . . . . . . . . . . . 168

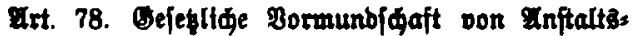
porftänben ober oon Deamten ber grmenver. maltung . . . . . . . . . . . . . 169

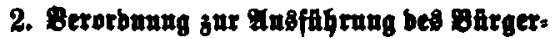
Itqen OSefeqbuqs. Bom 16. Soveruber 1899.

\&rt. 13. EheltglettBertlărung . . . . . . . 170

4rt. 14. Innafme an Rtnbes Statt . . . . . 171

3. Allgemetze Berfitgang vout 14.De. 1899171

4. Berfigung ves Juftizuinifters uns bes

Ertuifter bes 3mutu vom 28. Dlt. 1899174

III. Qubelle jux Estedaung bex

Gmpfungut/gatt . . . . 177

Gradregifter . . . . . . . . . . 190 
